\title{
SHOULDER ROTATOR CUFF BALANCE, STRENGTH AND ENDURANCE IN YOUNG SWIMMERS DURING A COMPETITIVE SEASON
}

\begin{abstract}
The purpose of this study was to analyse the effects of a competitive swim season on the strength, balance and endurance of shoulder rotator cuff muscles in young swimmers. A repeated-measures design was used with three measurements performed during the swim season. A swimmers group $(n=20)$ of young males with no dry-land training and a sedentary group $(n=16)$ of male students with the same characteristics (age, body mass, height and maturational state) were evaluated. In both groups, the peak torque of shoulder internal (IR) and external (ER) rotators was assessed during preseason, midseason (16 weeks), and postseason (32 weeks). Concentric action at $60^{\circ} \cdot \mathrm{s}^{-1}$ and $180^{\circ} \cdot \mathrm{s}^{-1}$ was measured using an isokinetic dynamometer. The ER/IR strength ratios and endurance ratios were also obtained. At $60^{\circ} \cdot \mathrm{s}^{-1}$, there were significant training effects in the IR strength and ER/IR ratio on both shoulders. This trend was the same throughout the competitive season. The same trend was present at $180^{\circ} \cdot \mathrm{s}^{-1}$ since the training effects are seen primarily in IR and ER/IR ratios. With respect to endurance ratios, within-group data were similar in ER and IR for both shoulders, with no significant differences between moments. However, betweengroup differences occurred mostly in the IR. Results suggest that a competitive swim season favours the increase of muscular imbalances in the shoulder rotators of young competitive swimmers, mainly due to increased levels of IR strength and endurance that are proportionally larger than those of their antagonists. A compensatory strength training program should be considered.
\end{abstract}

Key words: swimming; isokinetic strength; muscle balance; shoulder rotators. 